PROCEEDINGS OF THE

AMERICAN MATHEMATICAL SOCIETY

Volume 136, Number 1, January 2008, Pages 127-131

S 0002-9939(07)08916-2

Article electronically published on August 30, 2007

\title{
A LOWER BOUND FOR THE EQUILATERAL NUMBER OF NORMED SPACES
}

\author{
KONRAD J. SWANEPOEL AND RAFAEL VILLA
}

(Communicated by N. Tomczak-Jaegermann)

\begin{abstract}
We show that if the Banach-Mazur distance between an $n$-dimensional normed space $X$ and $\ell_{\infty}^{n}$ is at most $3 / 2$, then there exist $n+1$ equidistant points in $X$. By a well-known result of Alon and Milman, this implies that an arbitrary $n$-dimensional normed space admits at least $e^{c \sqrt{\log n}}$ equidistant points, where $c>0$ is an absolute constant. We also show that there exist $n$ equidistant points in spaces sufficiently close to $\ell_{p}^{n}, 1<p<\infty$.
\end{abstract}

\section{Notation}

Throughout the paper we use the same symbol $c$ for different absolute positive constants. Let $X$ denote a normed space of finite dimension $\operatorname{dim} X=n$. Let $e(X)$ denote the largest size of an equilateral set in $X$. As usual, the space $\ell_{p}^{n}, 1 \leq p<\infty$, is defined as $\mathbb{R}^{n}$ with the norm $\left\|\left(x_{1}, x_{2}, \ldots, x_{n}\right)\right\|_{p}=\left(\sum_{i=1}^{n}\left|x_{i}\right|^{p}\right)^{1 / p}$, and $\ell_{\infty}^{n}$ is $\mathbb{R}^{n}$ with the norm $\left\|\left(x_{1}, x_{2}, \ldots, x_{n}\right)\right\|_{\infty}=\max _{i}\left|x_{i}\right|$. The Banach-Mazur distance between two $n$-dimensional normed spaces is defined as $d(X, Y)=\inf \|T\|\left\|T^{-1}\right\|$, where the infimum is taken over all linear, invertible operators $T: X \rightarrow Y$. We say that $X$ is a $(1+\varepsilon)$-copy of $Y$ if $d(X, Y) \leq 1+\varepsilon$.

\section{The MAIN THEOREMS}

It is conjectured [4, 9, 11, 12, 17 that $e(X) \geq n+1$ for all $n$-dimensional normed spaces $X$. This is known for $n \leq 3$ [12] but open for $n \geq 4$. It is true for spaces sufficiently close to Euclidean:

Theorem 1 (Brass $[3$ and Dekster $[6])$. Let $X$ be an $n$-dimensional normed space with Banach-Mazur distance $d\left(X, \ell_{2}^{n}\right) \leq 1+\frac{1}{n}$. Then an equilateral set in $X$ of at most $n$ points can be extended to one of $n+1$ points. In particular, $e(X) \geq n+1$.

Combining this theorem with Schechtman's estimate [13, Theorem 3] in the Dvoretzky theorem [7, §4], the following general lower bound follows:

$$
e(X) \geq \frac{c \sqrt{\log n}}{\log \log n} .
$$

Received by the editors March 23, 2006 and, in revised form, September 1, 2006.

2000 Mathematics Subject Classification. Primary 46B04; Secondary 46B20, 52A21, 52C17.

This material is based upon work supported by the South African National Research Foundation under Grant number 2053752. The second author thanks the DGES grant BFM2003-01297 for financial support. Parts of this paper were written during a visit of the second author to the Department of Mathematical Sciences, University of South Africa, in January 2006.

(C) 2007 American Mathematical Society Reverts to public domain 28 years from publication 
(The previous best estimate in the Dvoretzky theorem, due to Gordon [8], gave $e(X) \geq c(\log n)^{1 / 3}$.) We improve this to the following:

Theorem A. For any $n$-dimensional normed space $X$ we have $e(X) \geq e^{c \sqrt{\log n}}$, where $c>0$ is an absolute constant.

The theoretical limit beyond which Dvoretzky's theorem cannot be improved in general (by considering $\ell_{\infty}^{n}$; see the discussion above Theorem 3 in [13]) would give only $e(X) \geq c \log n / \log \log n$, still worse than Theorem A Thus something more than Dvoretzky's theorem is needed. Indeed, the proof of Theorem $\mathrm{A}$ works by looking for large subspaces close to either $\ell_{2}^{k}$ or $\ell_{\infty}^{k}$. To this end we use the following theorem:

Theorem 2 (Alon and Milman 1]). Let $X$ be an $n$-dimensional normed space. Then for each $\varepsilon>0$ there exists $c \in(0,1)$ such that $X$ has a subspace of dimension $m \geq e^{c \sqrt{\log n}}$ that is $(1+\varepsilon)$-isomorphic to either $\ell_{2}^{m}$ or $\ell_{\infty}^{m}$.

If the second case occurs in the above theorem, i.e., if we find a $(1+\varepsilon)$-isomorphic copy of $\ell_{\infty}^{m}$ in $X$, then we need a result similar to Theorem 1 for spaces near $\ell_{\infty}^{n}$. This we provide as follows:

Theorem B. Let $X$ be an n-dimensional normed space with Banach-Mazur distance $d\left(X, \ell_{\infty}^{n}\right) \leq 3 / 2$. Then $e(X) \geq n+1$.

We can then choose $\varepsilon=1 / 2$ in Theorem 2 to obtain $e(X) \geq e^{c \sqrt{\log n}}$. On the other hand, if we find a $\left(1+\frac{1}{2}\right)$-isomorphic copy of $\ell_{2}^{m}$ where $m \geq e^{c \sqrt{\log n}}$, we cannot yet apply Theorem 1. We first have to find a $k$-dimensional subspace of the $m$-dimensional space that is $\left(1+\frac{1}{k}\right)$-isomorphic to $\ell_{2}^{k}$. This is guaranteed by the following:

Theorem 3 (Milman [10]). Let $X$ be an m-dimensional normed space and $0<$ $\varepsilon<1$. Then $X$ contains a subspace $Y$ of dimension $k \geq c \varepsilon^{2} m / d^{2}\left(X, \ell_{2}^{m}\right)$ such that $d\left(Y, \ell_{2}^{k}\right) \leq 1+\varepsilon$.

See also [7, Corollary 4.2.2]. Putting $\varepsilon=1 / k$ and $X$ the $m$-dimensional subspace that is $3 / 2$-isomorphic to $\ell_{2}^{m}$ into the above theorem, we obtain a subspace $Y$ of dimension $k>\mathrm{cm}^{1 / 3}$, and Theorem 1 then gives $e(X) \geq e(Y)>e^{c \sqrt{\log n}}$. To complete the proof of Theorem $\mathrm{A}$, it only remains to prove Theorem B

Proof of Theorem B. We use the Brouwer fixed point theorem [5, §14.3], as in Brass' proof of Theorem [1. Without loss of generality we may assume $X=\left(\mathbb{R}^{n},\|\cdot\|\right)$ and

$$
\|x\| \leq\|x\|_{\infty} \leq \frac{3}{2}\|x\| \quad \text { for all } x \in X .
$$

Let $I=\{(i, j): 1 \leq i<j \leq n+1\}$, with $|I|=n(n+1) / 2=N$. For $\varepsilon=\left(\varepsilon_{i, j}\right)_{(i, j) \in I} \in\left[0, \frac{1}{2}\right]^{N}$, let

$$
\begin{aligned}
p_{1}(\varepsilon) & =(-1,0, \ldots 0), \\
p_{j}(\varepsilon) & =\left(\varepsilon_{1, j}, \ldots \varepsilon_{j-1, j},-1,0, \ldots 0\right), \quad 2 \leq j \leq n-1, \\
p_{n}(\varepsilon) & =\left(\varepsilon_{1, n}, \ldots \varepsilon_{n-1, n},-1\right), \\
p_{n+1}(\varepsilon) & =\left(\varepsilon_{1, n+1}, \ldots \varepsilon_{n, n+1}\right) .
\end{aligned}
$$


For $1 \leq i<j \leq n$ we have $\left\|p_{i}(\varepsilon)-p_{j}(\varepsilon)\right\|_{\infty}=1+\varepsilon_{i, j}$. Define $\varphi:[0,1 / 2]^{N} \rightarrow$ $[0,1 / 2]^{N}$ by $\varphi_{i, j}(\varepsilon)=1+\varepsilon_{i, j}-\left\|p_{i}(\varepsilon)-p_{j}(\varepsilon)\right\|, 1 \leq i<j \leq n$. Note that

$$
\varphi_{i, j}(\varepsilon) \geq 1+\varepsilon_{i, j}-\left\|p_{i}-p_{j}\right\|_{\infty}=0
$$

and

$$
\varphi_{i, j}(\varepsilon) \leq 1+\varepsilon_{i, j}-\frac{2}{3}\left\|p_{i}-p_{j}\right\|_{\infty}=\frac{1}{3}\left(1+\varepsilon_{i, j}\right) \leq \frac{1}{2},
$$

so $\varphi$ is well defined. Brouwer now gives the existence of a point $\varepsilon^{\prime}=\left(\varepsilon_{i, j}^{\prime}\right) \in$ $[0,1 / 2]^{N}$ with $\varphi\left(\varepsilon^{\prime}\right)=\varepsilon^{\prime}$, which implies that $\left\|p_{i}\left(\varepsilon^{\prime}\right)-p_{j}\left(\varepsilon^{\prime}\right)\right\|=1$ for all $1 \leq i<$ $j \leq m$. We have obtained $n+1$ equilateral points.

\section{A generalization to $\ell_{p}^{n}$}

The following theorem partially generalizes Theorem B to all $\ell_{p}^{n}$ spaces with $1<p<\infty$.

Theorem C. For each $n>2$ and $p \in(1, \infty)$ there exists $R(p, n)>1$ such that for any $n$-dimensional normed space $X$ with Banach-Mazur distance $d\left(X, \ell_{p}^{n}\right) \leq R(p, n)$ we have $e(X) \geq n$. In fact,

$$
\begin{aligned}
R(p, n) & =\max _{\theta>0}\left(\frac{1+(1+\theta)^{p}}{2+(n-2) \theta^{p}}\right)^{1 / p} \\
& \sim 1+\frac{p-1}{2 p} n^{-\frac{1}{p-1}} \text { as } n \rightarrow \infty \text { with } p \text { fixed } .
\end{aligned}
$$

Proof. We follow the proof of Theorem B Assume $X=\left(\mathbb{R}^{n},\|\cdot\|\right)$ and

$$
\|x\| \leq\|x\|_{p} \leq R\|x\| \quad \text { for all } x \in X .
$$

Fix $\beta, \gamma>0$. Let $I=\{(i, j): 1 \leq i<j \leq n\}$, with $|I|=n(n-1) / 2=N$. For $\varepsilon=\left(\varepsilon_{i, j}\right)_{(i, j) \in I} \in[0, \beta]^{N}$, let

$$
\begin{aligned}
& p_{1}(\varepsilon)=(-\gamma, 0, \ldots 0), \\
& p_{j}(\varepsilon)=\left(\varepsilon_{1, j}, \ldots \varepsilon_{j-1, j},-\gamma, 0, \ldots 0\right), \quad 2 \leq j \leq n-1, \\
& p_{n}(\varepsilon)=\left(\varepsilon_{1, n}, \ldots \varepsilon_{n-1, n},-\gamma\right) .
\end{aligned}
$$

For $1 \leq i<j \leq n$ we have

$$
\left\|p_{j}-p_{i}\right\|_{p}^{p}=\sum_{k=1}^{i-1}\left|\varepsilon_{k, j}-\varepsilon_{k, i}\right|^{p}+\left(\varepsilon_{i, j}+\gamma\right)^{p}+\sum_{k=i+1}^{j} \varepsilon_{k, j}^{p}+\gamma^{p} .
$$

Define $\varphi:[0, \beta]^{N} \rightarrow[0, \beta]^{N}$ by $\varphi_{i, j}(\varepsilon)=1+\varepsilon_{i, j}-\left\|p_{i}-p_{j}\right\|$ for $1 \leq i<j \leq n$. On the one hand,

$$
\begin{aligned}
\varphi_{i, j}(\varepsilon) & \leq 1+\varepsilon_{i, j}-R^{-1}\left\|p_{i}-p_{j}\right\|_{p} \\
& \leq 1+\varepsilon_{i, j}-R^{-1}\left[\left(\gamma+\varepsilon_{i, j}\right)^{p}+\gamma^{p}\right]^{1 / p} .
\end{aligned}
$$

Taking into account that the latter is increasing with respect to $\varepsilon_{i, j}$, the inequality $\varepsilon_{i, j} \leq \beta$ implies that

$$
\varphi_{i, j}(\varepsilon) \leq 1+\beta-R^{-1}\left[(\gamma+\beta)^{p}+\gamma^{p}\right]^{1 / p} .
$$


Therefore, if $(\gamma+\beta)^{p}+\gamma^{p} \geq R^{p}$, then $\varphi_{i, j}(\varepsilon) \leq \beta$. On the other hand,

$$
\begin{aligned}
\varphi_{i, j}(\varepsilon) & \geq 1+\varepsilon_{i, j}-\left\|p_{i}-p_{j}\right\|_{p} \\
& \geq 1+\varepsilon_{i, j}-\left[(n-2) \beta^{p}+\left(\gamma+\varepsilon_{i, j}\right)^{p}+\gamma^{p}\right]^{1 / p} .
\end{aligned}
$$

Again the latter is increasing with respect to $\varepsilon_{i, j}$, so using $\varepsilon_{i, j} \geq 0$ we have

$$
\varphi_{i, j}(\varepsilon) \geq 1-\left[(n-2) \beta^{p}+2 \gamma^{p}\right]^{1 / p} .
$$

Then $\varphi_{i, j}\left(\varepsilon_{1}, \ldots \varepsilon_{m}\right) \geq 0$ would follow if $(n-2) \beta^{p}+2 \gamma^{p} \leq 1$. Subsequently, if

$$
(\gamma+\beta)^{p}+\gamma^{p} \geq R^{p} \quad \text { and } \quad(n-2) \beta^{p}+2 \gamma^{p} \leq 1,
$$

then $\varphi$ is well defined. Brouwer now gives a point $\varepsilon^{\prime}=\left(\varepsilon_{i, j}^{\prime}\right) \in[0, \beta]^{N}$ such that $\varphi\left(\varepsilon^{\prime}\right)=\varepsilon^{\prime}$, implying that the points $p_{1}\left(\varepsilon^{\prime}\right), \ldots p_{n}\left(\varepsilon^{\prime}\right)$ are equilateral.

Finally, to take the best choice for the parameters in (囷) we have to maximize the expression $(\gamma+\beta)^{p}+\gamma^{p}$ under the constraints $(n-2) \beta^{p}+2 \gamma^{p} \leq 1$ and $\beta, \gamma \geq 0$. Setting $\theta=\beta / \gamma$, we obtain

$$
R^{p}=\max _{\theta>0} \frac{1+(1+\theta)^{p}}{2+(n-2) \theta^{p}} .
$$

It is not difficult to see that for $\theta$ close to $n^{-1 /(p-1)}$, the right-hand side is $>1$ and $R-1 \sim \frac{p-1}{2 p} n^{-\frac{1}{p-1}}$.

\section{Concluding Remarks}

For $p=2$ the estimate in the above theorem is $d\left(X, \ell_{2}^{n}\right) \lesssim 1+\frac{1}{4 n}$, slightly worse than Theorem 1, However, we don't know how to obtain $n+1$ equidistant points as in Theorem B. It would also be interesting to know whether arbitrary equilateral sets of at most $n$ points in spaces near $\ell_{p}^{n}$ can be extended as in Theorem 1 . A different idea will be needed to extend the above theorem to the case $p=1$. See [16] for a survey on equilateral sets, as well as [2, 14, 15, for further results on equilateral sets in $\ell_{p}^{n}$.

\section{ACKNOWLEDGMent}

We thank the referee for helpful remarks leading to an improved paper.

\section{REFERENCES}

[1] N. Alon and V. D. Milman, Embedding of $l_{\infty}^{k}$ in finite-dimensional Banach spaces, Israel J. Math. 45 (1983), 265-280. MR720303 (85f:46027)

[2] N. Alon and P. Pudlák, Equilateral sets in $l_{p}^{n}$, Geom. Funct. Anal. 13 (2003), no. 3, 467-482. MR1995795 (2004h:46011)

[3] P. Brass, On equilateral simplices in normed spaces, Beiträge Algebra Geom. 40 (1999), no. 2, 303-307. MR.1720106 (2000i:52012)

[4] P. Brass, W. Moser, and J. Pach, Research problems in discrete geometry, Springer, New York, 2005. MR2163782(2006i:52001)

[5] A. Browder, Mathematical analysis: An introduction, Springer-Verlag New York, 1996. MR.1411675 (97g:00001)

[6] B. V. Dekster, Simplexes with prescribed edge lengths in Minkowski and Banach spaces, Acta Math. Hungar. 86 (2000), 343-358. MR1756257(2001b:52001)

[7] A. A. Giannopoulos and V. D. Milman, Euclidean structure in finite dimensional normed spaces, Handbook of the Geometry of Banach spaces (eds. W. B. Johnson and J. Lindenstrauss), Vol. 1, Elsevier, 2001, pp. 707-779. MR1863705 (2003b:46008)

[8] Y. Gordon, Some inequalities for Gaussian processes and applications, Israel J. Math. 50 (1985), 265-289. MR800188 (87f:60058) 
[9] B. Grünbaum, On a conjecture of H. Hadwiger, Pacific J. Math. 11 (1961), 215-219. MR0138044 (25:1492)

[10] V. D. Milman, New proof of the theorem of Dvoretzky on sections of convex bodies, Funct. Anal. Appl. 5 (1971), 28-37. MR0293374 (45:2451)

[11] F. Morgan, Minimal surfaces, crystals, shortest networks, and undergraduate research, Math. Intelligencer 14 (1992), no. 3, 37-44. MR.1184317 (93h:53012)

[12] C. M. Petty, Equilateral sets in Minkowski spaces, Proc. Amer. Math. Soc. 29 (1971), 369374. MR0275294 (43:1051)

[13] G. Schechtman, Two observations regarding embedding subsets of Euclidean spaces in normed spaces, Adv. Math. 200 (2006), 125-135. MR2199631 (2006j:46015)

[14] Cliff Smyth, Equilateral or 1-distance sets and Kusner's conjecture, manuscript, 2002.

[15] K. J. Swanepoel, A problem of Kusner on equilateral sets, Arch. Math. 83 (2004), 164-170. MR2104945 (2005i:52024)

[16] K. J. Swanepoel, Equilateral sets in finite-dimensional normed spaces, In: Seminar of Mathematical Analysis, eds. Daniel Girela Álvarez, Genaro López Acedo, Rafael Villa Caro, Secretariado de Publicationes, Universidad de Sevilla, Seville, 2004, pp. 195-237. MR2117069 (2005j:46009)

[17] A. C. Thompson, Minkowski geometry, Encyclopedia of Mathematics and its Applications, vol. 63, Cambridge University Press, Cambridge, 1996. MR1406315 (97f:52001)

Department of Mathematical Sciences, University of South Africa, PO Box 392, Pretoria 0003, South Africa

E-mail address: swanekj@unisa.ac.za

Departamento Análisis Matemático, Facultad de Matemáticas, Universidad de SeVilla, C/Tarfia, S/N, 41012 Sevilla, Spain

E-mail address: villa@us.es 